\title{
Immunoglobulin Heavy Chain Gene Rearrangement Studies in the Diagnosis of a Paediatric Conjunctival Lesion
}

\author{
Benjamin Cheuk Hung Lim ${ }^{a, b}$ Trent M. Sandercoe $e^{a, b}$ \\ aSt George Public Hospital, Kogarah, NSW, Australia; 'Sydney Eye Hospital, \\ Sydney, NSW, Australia
}

\section{Keywords}

Paediatric ophthalmology $\cdot$ Lymphoma $\cdot$ Immunopathology $\cdot$ Conjunctiva $\cdot$ Adnexa

\begin{abstract}
We present a case whereby standard immunohistochemistry and flow cytometry studies for a conjunctival biopsy were unable to reliably differentiate between the two distinct pathological processes of benign reactive lymphoid hyperplasia from conjunctival lymphoma. A tissue diagnosis was only able to be conclusively attained after the application of immunoglobulin heavy chain rearrangement studies to the specimen. This is unusual and to our knowledge has not been previously expressed in the literature. Hence, the use of these further molecular studies may have great potential clinical implications in helping resolve such diagnostic dilemmas.

(C) 2019 The Author(s)

Published by S. Karger AG, Basel
\end{abstract}

\section{Introduction}

Immunohistochemistry and flow cytometry are standard investigations utilised when analysing conjunctival biopsies [1,2]. However, when subsequent findings cannot definitively confirm a diagnosis, the role of further molecular analysis becomes increasingly important [1, 2]. We report a case whereby immunoglobulin heavy chain gene (IgH) rearrangement studies 
established the diagnosis of a conjunctival lesion from a paediatric patient as benign reactive lymphoid hyperplasia (BRLH).

\section{Case Report}

An otherwise healthy 12-year-old boy was referred to eye clinic for assessment of a rightsided conjunctival lesion. The lesion was noted 6 weeks prior, and steroid eye-drops were trialled for 2 weeks with minimal change in its characteristics. The patient was asymptomatic, denying any recent trauma, foreign body exposure or ophthalmic surgery. A salmon-coloured lesion was found within the right medial caruncle whilst a smaller lesion of similar appearance was incidentally noted in the left medial caruncle. The remainder of his ocular examination was normal.

An uncomplicated excision biopsy of the right conjunctival lesion was undertaken. From the recommended 1-month follow-up, the patient displayed no extra-ocular manifestations of lymphoma on physical examination [3]. A chest X-ray and serological investigations were also conducted with no abnormalities detected.

Flow cytometry of the specimen demonstrated an atypical lymphoid population of mostly mixed CD20-positive B cells and CD5-positive T cells, whilst histopathology displayed these atypical cells infiltrating germinal centres. Such features were suggestive of an extranodal marginal zone type lymphoma; however, a pattern of clonality supporting this diagnosis was not confirmed given the absence of both kappa and lambda light chain staining on immunohistochemistry. Further testing with IgH rearrangement studies demonstrated a polyclonal Bcell population which was consistent with BRLH.

Three months on, no additional visual or systemic symptoms had developed. The excision site was well healed; however, the left-sided lesion had increased in size. A left conjunctival excisional biopsy was conducted, undergoing the same immunohistochemistry and flow cytometry studies to confirm the presence of both kappa and lambda light chains and a subsequent diagnosis of BRLH.

\section{Discussion}

BRLH is characterised by highly concentrated lymphoid tissue with well-defined follicles and germinal centres [2-5]. Although uncommon in children, it is usually situated in the nasal fornix; such as this case [4]. The paediatric demographic has a higher incidence of mucosaassociated lymphoid tissue; thus, this patient might have developed eye-associated lymphoid tissue due to an antigenic hyper-stimulation reaction $[1,2]$. The use of steroid eye drops could have potentially masked the characteristics of the lesion, hence further confounding diagnosis. [3] Contamination or incorrect processing of the biopsy during initial histopathology and flow cytometry can produce inaccurate findings [5]. Since the proper methodology was conducted correctly for this case, this is unlikely. Eventual diagnosis of BRLH is reassuring given the very low risk of malignant transformation in children [3].

Immunohistochemistry and flow cytometry could not determine between a reactive lymphoid proliferative process or lymphoma for this patient. Normally one of either kappa or lambda light chains are found in lymphoma, whilst both light chains are present in BRLH, as seen in the left conjunctival biopsy $[1,5]$. Testing negative for both light chains from the initial 
right conjunctival biopsy is unusual and has not been previously documented from our literature review. Thus, the use of IgH rearrangement studies was critically important for differentiating between a polyclonal lymphoid population consistent with BRLH compared to monoclonality associated with lymphoma [1, 2, 5].

Absence of light chain staining from routine immunohistochemistry and flow cytometry of a conjunctival biopsy is unusual and complicates diagnosis. We report a case whereby IgH rearrangement studies established the diagnosis of BRLH from a conjunctival lesion in a paediatric patient.

\section{Acknowledgements}

We would like to thank Assoc. Prof. Robert Conway (Ophthalmology, Sydney Eye Hospital) and Dr. Michael Krivanek (Histopathology, Children's Hospital at Westmead) for their review and analysis of this case's pathology findings.

\section{Statement of Ethics}

Consent for publication of this case report has been obtained from the patient's guardian.

\section{Disclosure Statement}

The authors have no conflicts of interest to declare.

\section{Funding Sources}

No funding was obtained for this case report.

\section{Author Contributions}

Dr. Benjamin C.H. Lim (MBBS) and Dr. Trent M. Sandercoe (B. Med Sci Hons, MBBS, PHD, FRANZCO) are both acknowledged as the first authors of this case report, with equal contributions for conducting the literature review, liaising with relevant specialists, drafting, and confirmation of the final manuscript.

\section{References}

1 Verdijk RM. Lymphoproliferative tumors of the ocular adnexa. Asia Pac J Ophthalmol (Phila). 2017 MarApr;6(2):132-42.

2 Fukuhara J, Kase S, Noda M, Ishijima K, Yamamoto T, Ishida S. Conjunctival lymphoma arising from reactive lymphoid hyperplasia. World J Surg Oncol. 2012 Sep;10(1):194.

3 McLeod SD, Edward DP. Benign lymphoid hyperplasia of the conjunctiva in children. Arch Ophthalmol. 1999 Jun;117(6):832-5.

4 Shields CL, Chien JL, Surakiatchanukul T, Sioufi K, Lally SE, Shields JA. Conjunctival tumors: review of clinical features, risks, biomarkers, and outcomes - the 2017 J. Donald M. Gass lecture. Asia Pac J Ophthalmol (Phila). 2017 Mar-Apr;6(2):109-20. 
Lim and Sandercoe: Immunoglobulin Heavy Chain Gene Rearrangement Studies in the Diagnosis of a Paediatric Conjunctival Lesion

5 Beykin G, Pe'er J, Amir G, Frenkel S. Paediatric and adolescent elevated conjunctival lesions in the plical area: lymphoma or reactive lymphoid hyperplasia? Br J Ophthalmol. 2014 May;98(5):645-50. 